\title{
Applications of the Discrete Hodge Helmholtz Decomposition to Image and Video Processing
}

\author{
Biswaroop Palit ${ }^{1}$, Anup Basu ${ }^{2}$, and Mrinal K. Mandal ${ }^{1}$ \\ 1 Department of Electrical and Computer Engineering, University of Alberta, \\ Edmonton, AB, Canada T6G 2V4 \\ \{bpalit, mandal\}@ece.ualberta.ca \\ 2 Department of Computing Science, University of Alberta, \\ Edmonton, AB, Canada, T6G 2E8 \\ anup@cs.ualberta.ca
}

\begin{abstract}
The Discrete Hodge Helmholtz Decomposition (DHHD) is able to locate critical points in a vector field. We explore two novel applications of this technique to image processing problems, viz., hurricane tracking and fingerprint analysis. The eye of the hurricane represents a rotational center, which is shown to be robustly detected using DHHD. This is followed by an automatic segmentation and tracking of the hurricane eye, which does not require manual initializations. DHHD is also used for identification of reference points in fingerprints. The new technique for reference point detection is relatively insensitive to noise in the orientation field. The DHHD based method is shown to detect reference points correctly for $96.25 \%$ of the images in the database used.
\end{abstract}

\section{Introduction}

In this paper we consider two applications where the DHHD can be used for efficient image analysis. We implement an algorithm to detect and locate the eye of a hurricane accurately. Most of the existing techniques, like [1], use feature matching for the analysis of satellite images. As mentioned in [2], feature matching is suitable for smaller sized images, but is not efficient for satellite images which span a large spatial area. In addition, several set of features have to be extracted at different positions for the satellite images, complicating feature matching further. Our technique does not involve feature matching. Our method is automatic and does not require human intervention for initialization. [3] developed a method to estimate accurately the nonrigid motion and the cloud structure in a hurricane. Our algorithm can locate the hurricane eye with only an approximate representation of the motion field, obtained using the simple Block Matching Algorithm (BMA).

Fingerprint matching is a widely used biometric technique for personal identification. Two fingerprint images need to be registered before matching them. Thus robust reference point detection is a crucial step in fingerprint matching and classification. As defined in [4], the point with the maximum curvature on a convex ridge is an apt choice for a reference point. Application of DHHD on the fingerprint orientation field can then be used to locate the reference point in the fingerprint. Our algorithm is robust to the noise in the orientation field. 
The discrete Hodge-Helmholtz decomposition can be used to decompose a vector field into a sum of a curl free component, a divergence free component and a harmonic remainder. A field $\boldsymbol{\xi}$ is decomposed as:

$$
\boldsymbol{\xi}=\boldsymbol{\xi}_{C F}+\boldsymbol{\xi}_{D F}+\boldsymbol{\xi}_{H R}
$$

where $\boldsymbol{\xi}_{C F}$ is the curl free component, $\boldsymbol{\xi}_{D F}$ is the divergence free component and $\boldsymbol{\xi}_{H R}$ is the harmonic remainder. Associated with these different components, we have potential functions:

$$
\boldsymbol{\xi}_{C F}=\nabla E ; \boldsymbol{\xi}_{D F}=\nabla \times \boldsymbol{W}
$$

Here $E$ is a scalar potential and $\boldsymbol{W}$ a vector potential. The decomposed field, and its corresponding potential functions can be analyzed to predict singularities in the motion field. An implementation of the DHHD on regular triangular grids is developed in [5] and used for cardiac video analysis. We use a similar implementation for the DHHD.

Critical points like sources, sinks and rotational centers can be characterized on the basis of selected properties of the potential functions as follows:

- Point $\boldsymbol{p}$ is a source (velocity field diverges out of this point) if $E(\boldsymbol{p})$ is a minimum.

- Point $\boldsymbol{p}$ is a sink (field vectors converge to this point) if $E(\boldsymbol{p})$ is a maximum.

- A point $\boldsymbol{p}$ is an anticlockwise rotational center if $W(\boldsymbol{p})$ is a maximum.

- Point $\boldsymbol{p}$ is a clockwise rotational center if $W(\boldsymbol{p})$ is a minimum.

The subsequent discussion is organized as follows. Section 2 discusses the hurricane eye identification and tracking. Fingerprint analysis is described in Sect. 3. Some concluding remarks are outlined in Sect. 4

\section{Hurricane Tracking}

Automatic analysis of satellite images is an extremely important application in meteorology. We propose to use DHHD for detecting the eye of a hurricane, and then track it using a level set algorithm. The first task is to be able to predict the approximate location of the eye of the hurricane. This is followed by segmentation of the eye structure using the level set method. The proposed hurricane eye detection technique has several steps:

- Motion Detection: Motion is estimated using the BMA. We also tried motion estimation using an optical flow algorithm and an affine motion model. However, block matching is the simplest of the aforementioned methods, and provides satisfactory results, so we just present the results obtained using this particular algorithm. The field extracted is shown in Fig. 1(a)

- Motion Decomposition: DHHD is applied on the extracted field. As we are trying to detect a rotational center in this particular application, only the divergence free component of the field is of importance to us. The divergence free potential obtained is shown in Fig. 1(b) Since the video sequence shows the hurricane rotating in an anticlockwise direction, we get a distinct maxima. We need to locate the extremum points on this potential surface. 


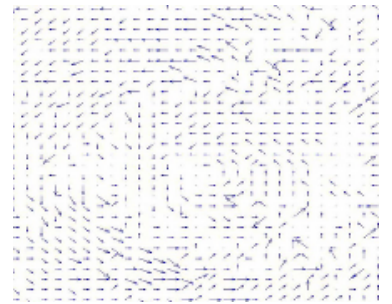

(a)

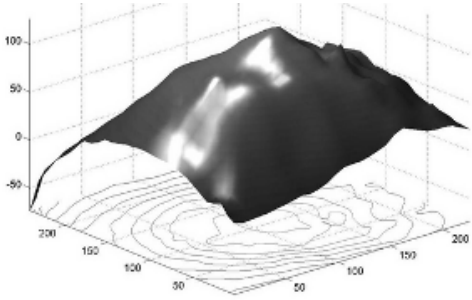

(b)

Fig. 1. (a) Motion field in a anticlockwise rotating hurricane sequence extracted using the BMA. (b) The divergence free potential function with a distinct maximum and corresponding contours.

- Locating the extremum point: To detect points of extrema in the potential functions, we check the eight adjacent grid points of each grid node. If the point is a maxima or a minima in this neighbourhood it is chosen as an extrema point. This point is classified as a critical point of the vector field according to the conditions described in Section 1

- Segmentation of the eye: We use the level set segmentation technique to segment out the eye from the hurricane image. The initial estimate for the location of the eye, obtained from the DHHD is used as the initial seed point. Details of the level set algorithm and its applications can be found in [6].

\subsection{Implementation and Experiments}

Image sequences of hurricane Luis (September 1995) and hurricane Isabel (September 2003), obtained from the NASA GOES satellites have been used for the experiments. Both sequences show the cloud mass rotating in an anticlockwise direction. The Hurricane Luis sequence has a smaller eye, compared to the Hurricane Isabel video sequence.

The motion field is estimated using the BMA with a block size of $8 \times 8$ and a search area of $16 \times 16$. This motion field is then fed into the DHHD subroutine and we extract the divergence free field and potential.

The initial estimate from DHHD turns out to be well within the eye of the hurricane. This extremum point indicated by the DHHD algorithm, and three other points in its neighborhood at a distance of 5 pixels each, are used as the initial points for the level set algorithm. The use of multiple initial seed points helps to speed up the level set algorithm. A narrow band fast marching implementation [6] of the level set method is used for reducing computational complexity. $N_{\text {iter }}$ iterations of the fast marching step give satisfactory segmentation of the eye. $N_{\text {iter }}$ depends on the size of the eye. For the video sequence Isabel, which has a bigger eye, $N_{i t e r}=550$ gives good results; whereas for the Hurricane Luis sequence, 300 iterations of the fast marching method are sufficient.

The consecutive frames in the sequences do not have a significant burst of motion. Hence, the location of the eye can be assumed to be approximately the same in consecutive frames. The continuity is exploited in the initialization of points for the eye segmentation in the next frame. The center point of the segmented eye in frame $N$, and two neighbouring points at a distance of 5 pixels are chosen as the initial seed points of frame $N+1$. Thus, we do not need to perform the DHHD step for every frame, which increases the computational speed. 


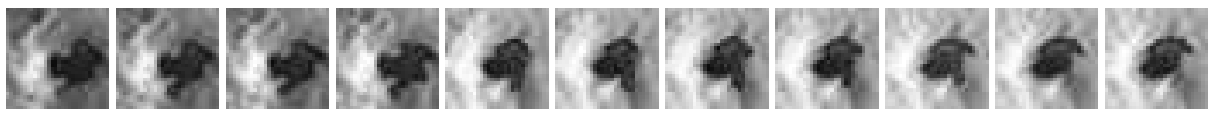

Fig. 2. Sequence of images from the Hurricane Luis sequence, with eye segmented

The DHHD algorithm has been implemented in Matlab, while the level set tracking is a $\mathrm{C}$ program. For the 83 frames of the Isabel sequence, we call the BMA and DHHD once and the level set program 83 times. The total time taken by BMA and DHHD combined is about 39 seconds while the level set program requires 649 seconds for its 83 calls. All these experiments have been performed on a Pentium $43.2 \mathrm{GHz}$ machine with $1 \mathrm{~GB}$ of RAM.

A series of segmented frames are shown in Fig. 2. As can be observed from these sequences, the performance of the tracking is accurate.

\section{Fingerprint Analysis}

Fingerprint recognition consists of reference point detection followed by feature extraction. Reference points are important for the registration of different fingerprint images [4]. We present a new algorithm for locating a reference point, which is defined as the point with maximum curvature in the ridge structure. The steps involved are described below.

- Preprocessing: The fingerprint image in consideration is preprocessed before analysis. An excellent source for fingerprint image enhancement is [7]. The fingerprint images are normalized. This process spreads the gray scale range of the given image over the entire spectrum of gray scales. Normalizing images makes it much easier to compare different images as all of them have the same range of gray scale. We use $\mu_{0}=100$ and $\sigma_{0}^{2}=100$ in the normalization algorithm from [7].

- Orientation field extraction: The orientation field is an estimate of the direction of the ridges and valleys in the fingerprint image. Since the valleys and ridges alternate periodically, the field direction can be estimated as the direction perpendicular to the intensity gradient. We follow the method described in [7] for extraction of the field.

The extracted motion field has a discontinuity of $\pi$ at certain locations. Such a discontinuity in the orientation field is observed in the circled part in Fig. 3(a), This will cause DHHD to fail as the direction of flow is reversed abruptly. To eliminate this discontinuity, the squared directional field is taken. The squared directional field is also used in [7] for eliminating the step of $\pi$ in the orientation field, before detecting singular points. This is shown in Fig. 3(b).

- DHHD: DHHD is now applied onto the squared directional field. We are trying to identify the point with the maximum curvature and hence only the divergence free field and potential are considered. The potential function should have an extrema at the point of maximum curvature, hence identifying the point. A potential surface is shown in Fig. 3(c) To locate the extremum point, we use the same method as discussed in the case of the hurricane eye application. An extracted reference point is shown in Fig.4(a), 


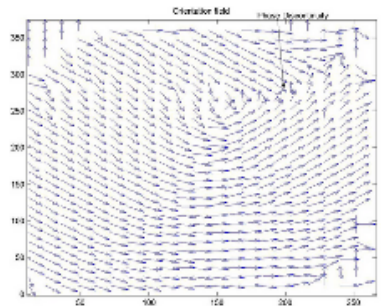

(a) Orientation Field

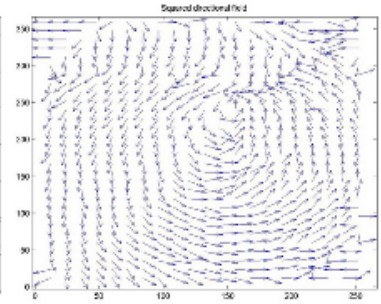

(b) Squared Orientation Field

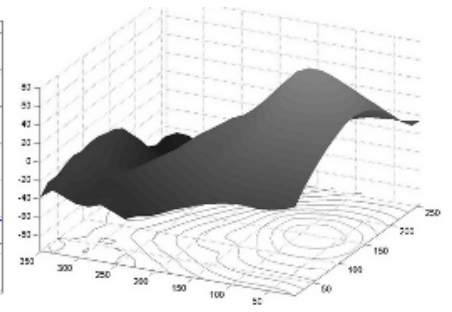

(c) Divergence free potential

Fig. 3. (a) Fingerprint orientation field (b) Squared orientation field (c) Potential function obtained from squared orientation field

\subsection{Experimental Results}

The FVC2000 database is used to test our algorithm [7]. This has 4 datasets, each with 8 different images of the same finger. We use the images of 50 fingers from each data set to test our methods. Each image is $500 \mathrm{dpi}$. Reference points detected for different images of the same finger are shown in Fig. 4(a), Using the same criteria as in [4], the distance between the DHHD predicted reference point and the manually detected reference point is considered as a measure of the performance of our algorithm. If the detected point is within 10 pixels of the manually detected reference point, the localization is considered accurate. If it is 20 pixels away, we assume detection with tolerable error. If the distance between the detected reference point and the manually predicted reference point is greater than 20 pixels, the detection is erroneous. For a 500 dpi image, 20 pixels correspond to about $1 \mathrm{~mm}$ in physical distance.

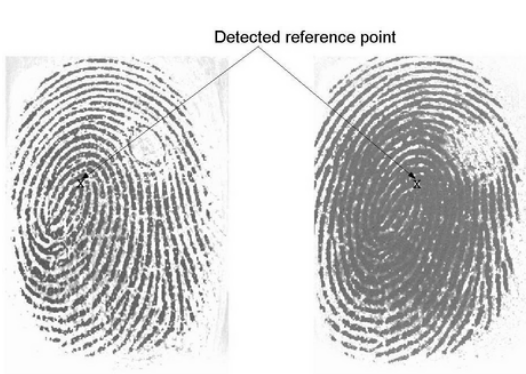

(a)

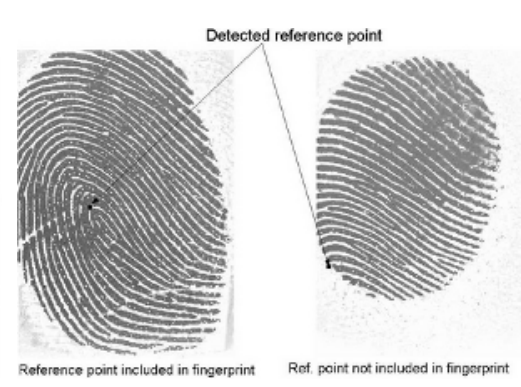

(b)

Fig. 4. (a) Reference point identified in two different images of the same finger (b) Images of the same finger with and without reference point, and the detected reference points

Table 1. Distance between reference point predicted by DHHD and point predicted manually

\begin{tabular}{|c|c|c|c|}
\hline Distance (pixels) & Number of fingerprints & Percentage & Results from [4] \\
\hline$\leq 10$ & 1355 & 84.69 & 81.07 \\
\hline$>10$ and $\leq 20$ & 185 & 11.56 & 13.72 \\
\hline$>20$ & 60 & 3.75 & 5.21 \\
\hline
\end{tabular}


There are some image sets where the reference point is not included in the fingerprint. Using the criteria described in [4], if the reference point is detected to be at the border as shown in Fig. 4(b), it is considered to be a correct detection. If it is elsewhere a faulty detection is reported. The results are shown in Table 1 . We get a correct detection in $96.25 \%$ of the cases, which is better than the $94.79 \%$ correct detection reported in $[4]$.

The algorithm has been implemented in Matlab. The entire algorithm (time includes preprocessing, field extraction and DHHD) takes 3.1 seconds on a $3.2 \mathrm{GHz}$ Pentium 4 $3.2 \mathrm{Ghz}$ machine with $1 \mathrm{~GB}$ of memory.

\section{Conclusions}

We applied the DHHD technique to two problems in image processing - hurricane tracking and fingerprint reference point identification. In the hurricane tracking application, motion between two consecutive frames was estimated using a block matching algorithm and then DHHD was used to identify the center of rotation (the hurricane eye). The estimate of the eye was then used to initialize a level set algorithm which tracked the eye over consecutive video frames. Thus, an automated system for hurricane eye tracking was developed. In the second application, DHHD is shown to be able to identify reference points in fingerprints. First the orientation field is extracted from the fingerprint image, after which DHHD is applied to the extracted field for critical point identification (the point with maximum curvature).

\section{References}

1. Mukherjee, D., Acton, S.: Cloud tracking by scale space classification. IEEE Transactions on Geoscience and Remote Sensing 40 (2002) 405-415

2. Bretschneider, T., Yikun, L.: On the problems of locally defined content vectors in image databases for large images. In: Proceedings of the International Conference on Information Communications and Signal Processing IEEE PCM. Volume 3. (2003) 1604-1608

3. Zhou, L., Kambhamettu, C., Goldgof, D., Palaniappan, K., Hasler, A.: Tracking nonrigid motion and structure from 2D satellite cloud images without correspondences. IEEE Transactions on Pattern Analysis and Machine Intelligence 23 (2001)

4. Jiang, X., Liu, M., Kot, A.: Reference point detection for fingerprint recognition. In: Proceedings of the 17th International Conference on Pattern Recognition, ICPR 2004. Volume 1. (2004) 540-543

5. Guo, Q., Mandal, M., Li, M.: Efficient hodge-helmholtz decomposition of motion fields. Pattern Recognition Letters 26 (2005) 493-501

6. Sethian, J.A.: Level Set Methods:Evolving Interfaces in Geometry,Fluid Mechanics, Computer Vision and Material Science. Cambridge University Press (1996)

7. Jain, A.K., Maltoni, D.: Handbook of Fingerprint Recognition. Springer-Verlag New York, Inc., Secaucus, NJ, USA (2003) 